\title{
Educational program on fatigue for brain tumor patients: possibility strategy?
}

\author{
Programa educativo para fadiga em pacientes com tumor cerebral: possibilidade de \\ estratégia?
}

Marcela dos Reis Bigatão', Fernanda Maris Peria², Daniela P. C. Tirapelli', Carlos Gilberto Carlotti Junior ${ }^{1}$

\begin{abstract}
Objective: To evaluate the effectiveness of an educational program on improvement of fatigue and quality of life of patients with high-grade glioma during radiotherapy and chemotherapy treatment. Method: This is a longitudinal, experimental study. Twenty-three patients with high-grade glioma were randomly assigned to one of two groups. Both groups completed the Functional Assessment of Cancer Therapy: Fatigue questionnaire and the Beck Depression Inventory, and one of the groups received the educational intervention. Results and Conclusions: The groups did not show any change in quality of life and fatigue in this study, for this reason, the educational program did not present any significant difference. However, there was a significant difference in depressive symptoms during the educational program showing positive evidence for its applicability.
\end{abstract}

Keywords: glioma, fatigue, depression, quality of life, health education.

\section{RESUMO}

Objetivo: Verificar a efetividade de um programa educativo na melhora da fadiga e dos sintomas depressivos em pacientes com glioma de alto grau durante o tratamento com radioterapia e quimioterapia. Método: Trata-se de estudo longitudinal e experimental. Foram incluídos 23 pacientes com glioma de alto grau e divididos aleatoriamente em 2 grupos. Os dois grupos responderam os questionários Functional Assessment of Cancer Therapy: Fatigue e Inventário de Depressão de Beck, porém somente um foi inserido ao programa educativo. Resultados e Conclusões: Os grupos não apresentaram alteração na fadiga ao longo desse estudo, assim o programa educativo não mostrou diferença significativa, porém nos sintomas depressivos, o programa educativo trouxe diferença quando estava sendo realizado mostrando evidências positivas para sua aplicabilidade.

Palavras-chave: gliomas, fadiga, depressão, qualidade de vida, educação em saúde.

Fatigue is recognized as one of the most common and distressing adverse effects of cancer disease and treatment ${ }^{1}$. For Bower ${ }^{1}$ and Raff et al. ${ }^{2}$, the clinical symptom of fatigue may include generalized weakness, decreased mental concentration, insomnia or hypersomnia, and emotional change that cause significant impairment to the global quality of life of cancer patients during and after treatment.

The pathogenesis of the relationship between cancer and fatigue has not been elucidated, but physiological, biochemical and psychological disturbances seem to be involved. Due to its multifactorial nature, several interventions in the management of cancer-related fatigue have been investigated ${ }^{2}$.
In a review study, Horneber et al. ${ }^{3}$ highlight some of the physiopathological factors of fatigue, including inflammatory cytokine dysregulation, changes in hypothalamic regulator circuits and serotonergic system in the central nervous system (CNS), and disturbances in circadian and sleep-wake rhythm, gene polymorphisms of regulatory proteins involved in oxidative phosphorylation, B-cell signal transduction, proinflammatory cytokine expression and catecholamine metabolism.

Some authors have investigated the frequency of fatigue in patients with glioblastoma and showed that these patients are commonly affected by this symptom, suggesting that other factors related to radiotherapy and/or chemotherapy, such as depression and tumor site, may have a significant impact ${ }^{4}$.

${ }^{1}$ Universidade de São Paulo, Faculdade de Medicina de Ribeirão Preto, Departamento de Cirurgia e Anatomia, Ribeirao Preto SP, Brazil;

¿Universidade de São Paulo, Faculdade de Medicina de Ribeirão Preto, Departamento de Oncologia, Ribeirao Preto SP, Brazil.

Correspondence: Marcela dos Reis Bigatão; Rua Erno Toth, 20;13272-081 Valinhos SP, Brasil. E-mail: marcelarb@usp.br

Conflict of interest: There is no conflict of interest to declare.

Received 10 July 2015; Received in final form 21 September 2015; Accepted 15 October 2015. 
Fatigue is reported by more than $80 \%$ of patients with primary brain tumor during radiotherapy ${ }^{5}$.

Gliomas are CNS tumors derived from glial cells, and comprise approximately $40 \%-60 \%$ of all CNS primary tumors, being the most common among adults ${ }^{6}$.

Biological characteristics of glioblastomas include rapid growth, which frequently evolve into increased intracranial pressure. Depending on its exact location, glioblastomas may cause neurological deficits, including slow thinking, ataxia, changed behavior, dizziness, motor deficits, visual impairment (blurred vision, diplopia), epilepsy, recurrent syncope, and in more severe cases, severe sleepiness and coma ${ }^{7}$. According to the literature, the survival time of patients with glioblastoma multiforme is $10-24$ months. The usual treatment is surgery, followed by radiotherapy and chemotherapy in some cases. In 2005, temozolomide (TMZ) was approved by the Food and Drugs Administration (FDA) as a first-line chemotherapeutic treatment for glioblastoma. Radiotherapy plus concomitant and adjuvant temozolomide have demonstrated meaningful clinical results, with statistically significant survival advantage and minimal toxicity ${ }^{8}$.

There is no gold-standard for fatigue treatment, possibly due to its multifactorial etiology and lack of knowledge of underlying mechanisms. However, some of the pharmacological or non-pharmacological approaches have been found to contribute to reduce cancer-related fatigue.

The most frequently used non-pharmacological approaches are physical activity (exercise), psychosocial interventions, including educational interventions, management of work and rest times, and relaxation and attention techniques ${ }^{1,3,9}$.

Among the psychosocial interventions, which include education, counseling, and support groups, the educational therapy and the cognitive-behavioral therapy have shown significant benefits to patients ${ }^{9}$.

Educational therapies aim to provide general information about fatigue and to introduce new concepts regarding energy conservation, self-care, and confrontation techniques ${ }^{9}$.

Energy conservation is defined as an individualized, intentional planning aimed at preventing to prevent a decrease in patient's energy. Strategies for energy conservation include identification of priorities, delegation of tasks, avoidance of unnecessary ambulation, and scheduling activities that require high-energy use during periods of peak energy ${ }^{10}$.

This study is aimed at identifying fatigue in patients with high-grade glioma during radiotherapy and chemotherapy, and evaluating the effectiveness of an educational program in terms of fatigue and quality of life.

\section{METHOD}

Twenty-three patients (aged 55 years [29-85 years]) with a diagnosis of high-grade glioma, initiating treatment at the oncologic neurosurgery outpatient clinic of the General Hospital of Ribeirão
Preto Medical School, University of São Paulo (HCFRMP-USP) and at the General Hospital of the University of Campinas were included in the study. This was a quantitative, longitudinal, experimental study, which was approved by the Ethics Committee of HCFRMP-USP (process number 9242/2011).

Subjects were assigned to one of two groups. In the first group (group I, $\mathrm{n}=10,5$ men), patients completed the Functional Assessment of Cancer Therapy-Fatigue (FACIT-F) and the Brazilian validated version of the Beck Depression Inventory (BDI) at three time points: before radiotherapy administered in combination with TMZ according to local and international treatment protocols, one month after concurrent radiotherapy with TMZ, and after the third cycle of TMZ alone, corresponding to a mean of 6 months of follow-up. There were four dropouts because of clinical reasons or death.

In the second group (group II, $\mathrm{n}=13,7$ men), the subjects underwent the same protocol as group I, followed by an educational program, in which patients received an informative leaflet (Figure 1) and preventive information about fatigue. Printed materials allow the patients to read them afterwards, and be used as guidelines. Four patients were excluded because of clinical reasons or death, and $5(56 \%)$ patients adhered to the program.

The leaflet aimed at promoting health education regarding fatigue. The content of the leaflet focused a non-pharmacological approach of fatigue, and was designed based on four types of contents (references to current literature on the subject): informational, energy conservation, physical exercise (walking) and sleep management.

At the time when patients received the leaflets, the investigator read it with the patient and gave them individual instructions on how to perform daily routine activities (bathing, clothing, mobility, eating, personal hygiene) using as little energy as possible. In addition, all patients attended seven occupational therapy sessions, in which they reported their daily activities and difficulties to perform them. The first session was carried out before the administration of radiotherapy combined with TMZ, the five following sessions were conducted during this treatment, the sixth session at one month after radiotherapy, and the last session at the third cycle of exclusive chemotherapy.

\section{Data analysis}

A mixed-effects regression model was used for comparisons between groups and time points. The analysis was performed using Proc MIXED of SAS 9.2 software.

\section{RESULTS}

\section{FACIT- F}

The FACIT-F is a 40-item questionnaire, composed by the 27-item Functional Assessment of Cancer Therapy-General (FACIT-G) and the 13-item FACIT-F. Higher FACIT-F scores indicate higher quality of life (Figure 2). 


\section{Exercises}

Light to moderate exercises include walking, treadmill, stationary cycling, hydrotherapy, light weight training, stretching and relaxation. The exercises should be done 3-5 times a week, preferentially during the daytime, for at least 30 minutes.

If you cannot do the exercises for 30 minutes uninterruptedly, in the beginning, you may divide it into shorter sessions throughout the day. For example, 10 minutes in the morning, 10 minutes in the early afternoon and 10 minutes in the late afternoon.

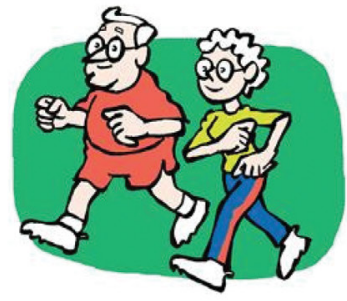

\section{Keep a balance between exercise and rest!!!}

Research Project on Fatigue

\section{INFORMATION ABOUT FATIGUE}

If you have any questions, please contact : Investigator : Marcela dos Reis Bigatão Occupational therapist HC FMRP-USP PhD student, Department of Surgery Program FMRP-USP

Supervisors: Professor Carlos Gilberto Carlloti Professor Carmem Silvia Passos Lima

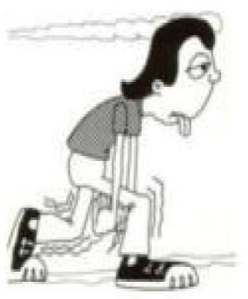

\section{FATIGUE}

\section{What is fatigue?}

Fatigue is a feeling of profound tiredness which is not alleviated by rest, and has a negative impact on physical, psychological and social functions, drastically reducing the quality of life.

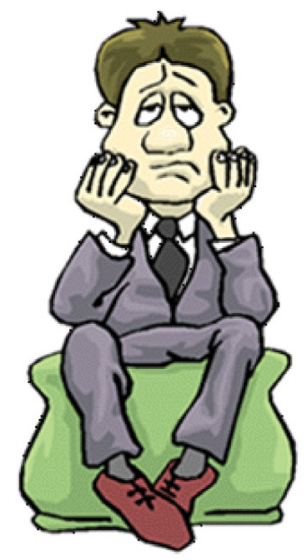

\section{What is the cause of fatigue?}

Fatigue may be caused by the disease, radiotherapy and chemotherapy treatment, anemia, lack of sleep, mood swing, among other causes.

\section{How can I prevent and/or alleviate fatigue?}

\section{Energy conservation}

- Planning is the key word when the individual should eliminate unnecessary activities and organize his living and working environments, as well as the time spent in daily life activities.

- Bathing: take the bath/shower at peak energy times, for example in the morning. If necessary, use a shower chair and long handled shower sponges

- Dressing : lay out your clothes and bring them to the bathroom. Sit down to get dressed, put on the pants first and then the shirt.

- Doing the dishes, cooking, laundry : schedule household tasks, avoid doing all at once. Prepare your meals in stages, assemble the ingredients before starting to cook. Let dishes air dry without wiping.
- Sweeping, mopping, cleaning : clean one room at a time, and take rest breaks. Use the mop instead of the broom. Get all the items you will need before start. - Arranging your environment : store items most often used in easy to reach areas.

\section{Sleeping}

Improving your sleep: avoid long naps and avoid napping too late in the day. Set a time to go to bed and a time to get up. Arrange a quiet environment before going to bed.

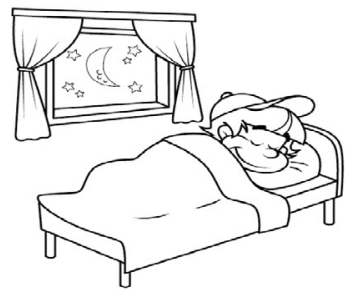


A
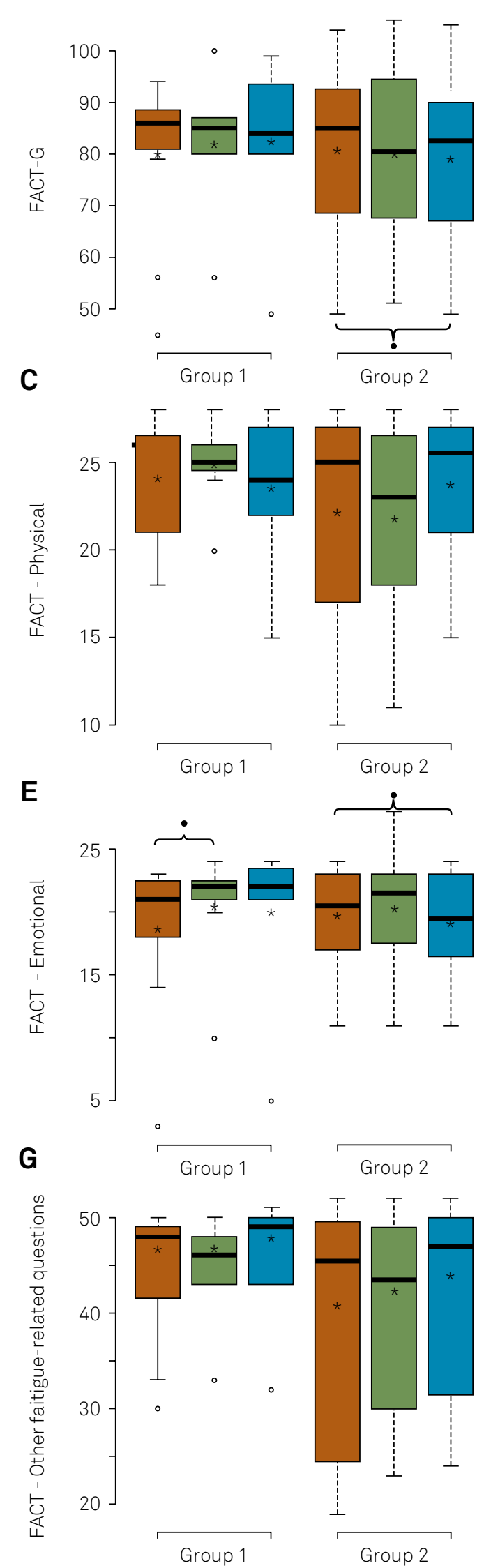

B
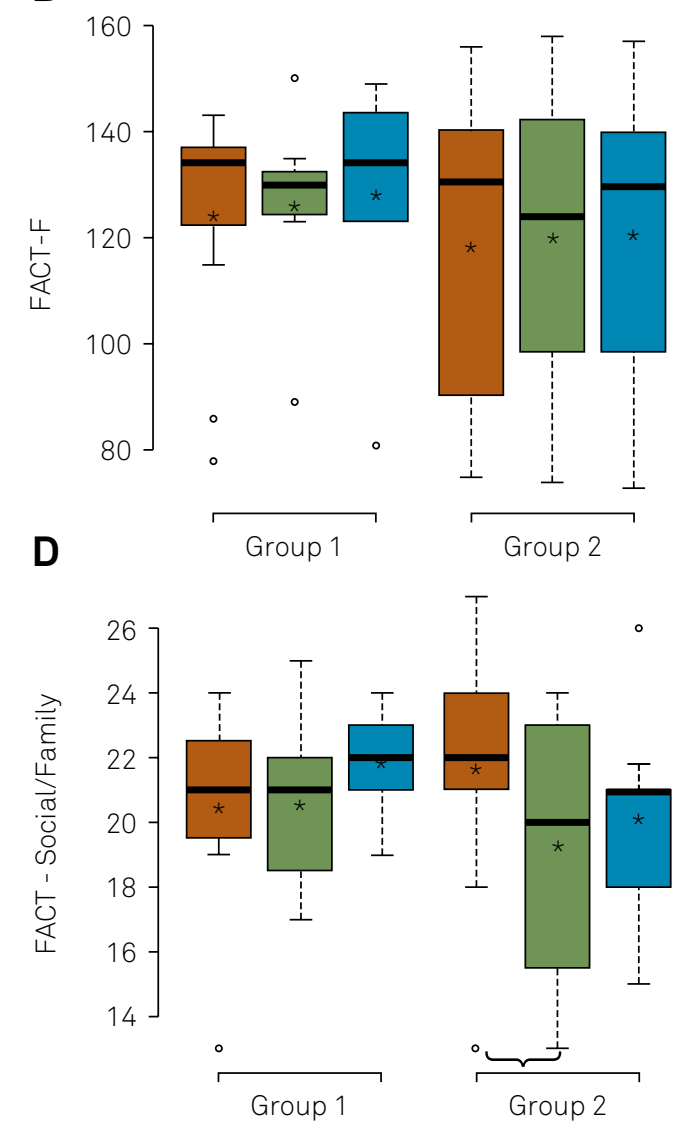

$\mathbf{F}$

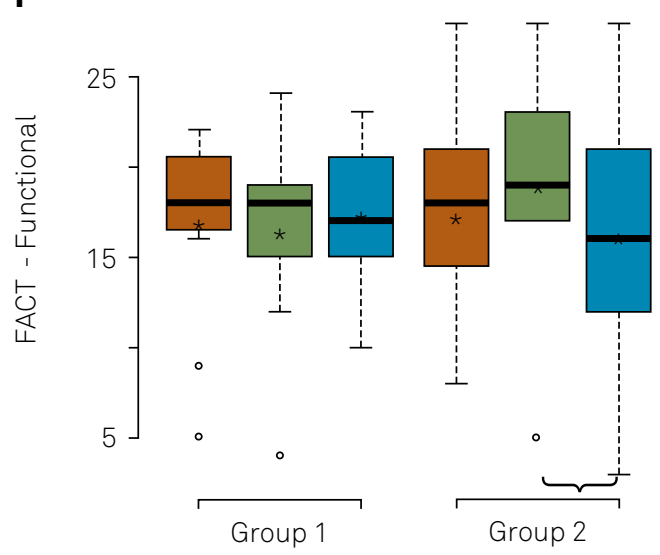

Time

$\square 1 \square 2 \square 3$

Figure 2. Comparisons between-group (groups I and II) and intra-groups at three time points (post-surgery, one month after radiotherapy, and three months after the TMZ chemotherapy) of FACIT-G and FACIT-F (physical well-being, social/family well-being, emotional well-being, functional well-being and other fatigue-related questions) scores using mixed-effects regression model. 
In group I, a statistically significant difference in emotional well-being was observed between the first (post-surgery) and the second (post-radiotherapy) time points.

In group II (educational program), there was a statistically significant difference $(\mathrm{p}<0.04)$ in FACT-G scores between the first valuation (post-surgery) and the third time point (three months after adjuvant chemotherapy). In addition, there were significant differences in the domain social/family well-being $(\mathrm{p}<0.04)$ between the three time points, in the domain emotional well-being $(\mathrm{p}<0.01)$ between the third (three months after chemotherapy) and the first time points (post-surgery), and in the domain functional well-being $(\mathrm{p}<0.04)$ between the third and the second time points (post-radiotherapy).

\section{Beck depression inventory}

The Beck depression inventory is a 21-item self-report inventory for measuring the severity of depression symptoms. The standard cut-offs of this test are: 0-9 indicates minimal depression; 10-18 indicates mild depression; 19-29 indicates moderate depression, and 30-63 indicates severe depression. Higher scores indicate more severe depression symptoms.

In the intra-group comparisons (Figure 3), a statistically significant difference was observed between the first and the third time points $(\mathrm{p}<0.04)$, and between the second and the third time points $(\mathrm{p}<004)$ in group I, whereas in group II, a statistically significant difference was observed between the first and the second time points $(\mathrm{p}<0.03)$.

\section{DISCUSSION}

In the present study, the subjects were randomly assigned to one of two groups, one group receiving radiotherapy and chemotherapy only (group I), and the other receiving radiotherapy, chemotherapy and educational intervention

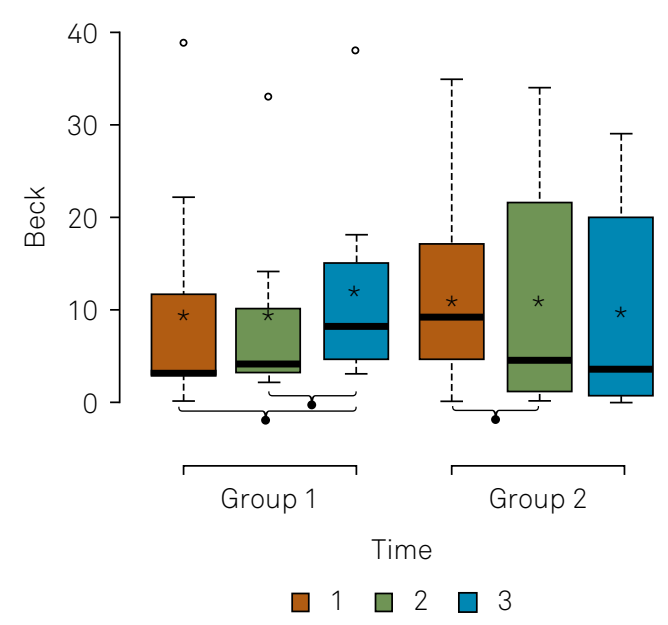

Figure 3. Comparisons between-group (groups I and II) and intra-groups at three time points (post-surgery, one montha after radiotherapy, and three months after the $T M Z$ chemotherapy) of Beck Depression Inventory scores. (group II). In the group II, most patients were men, which is in accordance with the frequency of high-grade glioma, relatively higher among men than women (3:1 proportion) ${ }^{6}$.

The educational program directed for fatigue management in patients with high-grade glioma in group II did not show a statistically significant effect on fatigue or quality of life, as compared to group I.

In intra-group comparisons, the FACT-G, used to measure health-related quality of life in cancer patients, was significantly different between the post-operative period and at three months after adjuvant chemotherapy, indicating a decrease in quality of life. This finding corroborates with that previously reported by a study aiming at evaluating the quality of life of patients with malignant brain tumor after combined treatments in comparison with patients with other chronic neurological conditions, and presenting the relationship between quality of life and clinical, pathological, affective and cognitive factors ${ }^{11}$. The author showed that quality of life is significantly associated with depression, state of anxiety and performance status, and physical and cognitive performances seem to play a major role in determining quality of life in brain tumor patients. In a recent study ${ }^{12}$ evaluating subjective aspects of quality of life and their relation to the type of brain tumor and stage of disease, quality of life was found to encompass different subjective aspects, which vary in relation to disease stage, and clinical worsening. Additionally, personal facets are independent on disease progression and treatment, suggesting the influence of individual resources ${ }^{12}$.

Intra-group comparisons of FACT-F revealed improved emotional well-being in group I after radiotherapy $(p<0.02)$ as compared to previous time point. Group II showed lower social/family well-being $(\mathrm{p}<0.04)$ one month after radiotherapy (second time point 2) as compared to post-surgery ( first time point). The emotional well-being category was significantly lower $(\mathrm{p}<0.01)$ at three months after adjuvant chemotherapy (third time point) as compared to post-surgery (second time point), and functional well-being was significantly lower $(\mathrm{p}<0.04)$ at three months after adjuvant chemotherapy as compared to post-radiotherapy.

As previously described, in the intervention group (group II), a decrease in social/family well-being, emotional well-being and functional well-being, and reduction in global quality of life was observed. Since patients received the educational intervention after surgery ( first time point) and after radiotherapy (second time point), we can suggest that the emotional and functional categories decreased as the frequency of sessions also decreased. Therefore, we may consider that weekly sessions would be appropriate to provide adequate support and opportunity for patients to receive energy conservation education and to discuss difficulties in performing daily activities.

According to Paulo Freire ${ }^{13}$, awareness brings out one's sense and responsibility of "announcers" or "whistleblowers", and opens up the possibility to disclose the reality. Critical consciousness of education focusing on awareness, change 
and freedom requires proximity of professionals to the population. In comprehensive, individualized approaches, health education is delivered to a particular person, whose experiences and characteristics may increase the effectiveness of preventive and therapeutic strategies. In this study, the informative leaflet was used with the aim of providing written instructions and be used as a guideline by the patients. However, differently from an impartial approach, our educational program also encompassed individualized meetings/sessions, which showed a positive effect on patients' well-being during radiotherapy.

Radiotherapy-related fatigue has been frequently reported by patients with primary brain tumor, although some authors argue that fatigue in patients with glioblastoma may not be a complication of the therapy (radiotherapy or chemotherapy), since the prevalence of fatigue is high even before the therapy ${ }^{4}$. Therefore, radio-chemotherapy toxicity may only partly contribute to therapy-related fatigue.

Regarding to the BDI, which measures the symptoms of depression, no statistically significant differences were observed in the BDI scores between the groups. However, in intra-group comparisons, a statistically significant difference ( $p<0.04$ ) was observed between post-surgery ( first time point) and three months after adjuvant chemotherapy (third time point), and between one month after radiotherapy (second time point) and three months after chemotherapy (third time point) $(\mathrm{p}<0.04)$, indicating an aggravation of the symptoms in group I. On the other hand, in group II, a statistically significant difference $(\mathrm{p}<0.03)$ was observed between postsurgery (first time point) and one month after radiotherapy (second time point), indicating a reduction in depression symptoms. This finding supports the importance of the occupational therapy sessions as an opportunity for teaching and listening. Listening is considered an important tool to empower the individual to adapt to and deal with his new condition, often full of limitations and changes.

Depression was associated with a decline in physical function, cognitive deficit, and quality of life among brain tumor patients ${ }^{14}$. In addition, patients with glioma are at higher risk of phychiatric disorders during the period of diagnosis ${ }^{15,16}$. The prevalence of depression in glioma patients is estimated to vary from $0 \%$ to $93 \%{ }^{14}$.

The groups did not show any change in quality of life and fatigue in this study, for this reason, the educational program did not present any significant difference. However, there was a significant difference in depressive symptoms during the educational program showing positive evidence for its applicability. Therefore, this study provides contributions to understand the relationship between fatigue and depressive symptoms. It also brings the possibility of non-pharmacological intervention for the treatment of fatigue in patients with brain tumor.

\section{References}

1. Bower JE. Cancer-related fatigue-mechanisms, risk factors, and treatments. Nat Rev Clin Oncol. 2014;11(10):597-609. doi:10.1038/nrclinonc. 2014.127

2. Raaf PJ, Klerk C, Timman R, Busschbach JJV, Oldenmenger WH, Rijt CCD. Systematic monitoring and treatment of physical symptoms to alleviate fatigue in patients with advanced cancer: a randomized controlled trial. J Clin Oncol. 2013;31(6):716-23. doi:10.1200/JCO.2012.44.4216

3. Horneber M, Fischer I, Dimeo F, Rüffer JU, Weis J. Cancer-related fatigue: epidemiology, pathogenesis, diagnosis, and treatment. Dtsch Arztebl Int. 2012;109(9):161-72. doi:10.3238/arztebl.2012.0161

4. Valko PO, Siddique A, Linsenmeier C, Zaugg K, Held U, Hofer S. Prevalence and predictors of fatigue in glioblastoma: a prospective study. Neuro-oncol. 2015;17(2): 274-81. doi:10.1093/neuonc/nou127

5. Armstrong TS, Gilbert MR. Practical strategies for management of fatigue and sleep disorders in people with brain tumors. Neuro-oncol. 2012;14(suppl 4):i65-72. doi:10.1093/neuonc/nos210

6. Instituto Nacional de Câncer José Alencar Gomes da Silva. Estatística do câncer: vigilância e de fatores de risco. Brasília, DF: Instituto Nacional do Câncer; 2014 [cited 2015 Jan 12] Available from: http://www.inca.gov.br/estimativa/2014/

7. Lakhan SE, Harle L. Difficult diagnosis of brainstem glioblastoma multiforme in a woman: a case report and review of the literature. J Med Case Reports. 2009;3(1):87. doi:10.1186/1752-1947-3-87

8. Stupp R, Mason WP, Bent MJ, Weller M, Fisher B, Taphoorn MJB, Belanger K et al. Radiotherapy plus concomitant and adjuvant temozolomide for glioblastoma. N Engl J Med. 2005;352(10):987-96. doi:10.1056/NEJMoa043330
9. Pachman DR, Price KA, Carey EC. Nonpharmacologic approach to fatigue in patients with cancer. Cancer J. 2014;20(5):313-8. doi:10.1097/PP0.0000000000000064

10. Barsevick AM, Whitmer K, Sweeney C, Nail LM. A pilot study examining energy conservation for cancer treatment-related fatigue. Cancer Nurs. 2002;25(5):333-41. doi:10.1097/00002820-200210000-00001

11. Giovagnoli, A. Quality of life in patients with stable disease after surgery, radiotherapy, and chemotherapy for malignant brain tumour. J Neurol Neurosurg Psychiatry. 1999;67(3):358-63. doi:10.1136/jnnp.67.3.358

12. Giovagnoli AR, Meneses RF, Silvani A, Milanesi I, Fariselli L, Salmaggi A et al. Quality of life and brain tumors: what beyond the clinical burden? J Neurol. 2014;261(5):894-904. doi:10.1007/s00415-014-7273-3

13. Freire P. Conscientização: teoria e prática da libertação; uma introdução ao pensamento de Paulo Freire. São Paulo: Moraes; 1980.

14. Rooney AG, Carson A, Grant R. Depression in cerebral glioma patients: a systematic review of observational studies. J Natl Cancer Inst. 2011;103(1):61-76. doi:10.1093/jnci/djq458

15. Dalton SO, Laursen TM, Ross L, Mortensen PB, Johansen C. Risk for hospitalization with depression after a cancer diagnosis: a nationwide, population-based study of cancer patients in Denmark from 1973 to 2003. J Clin Oncol. 2009;27(9):1440-5. doi:10.1200/JC0.2008.20.5526

16. Benros ME, Laursen TM, Dalton SO, Mortensen PB. Psychiatric disorder as a first manifestation of cancer: a 10-year population-based study. Int J Cancer. 2009;124:2917-22. doi:10.1002/ijc.24274 